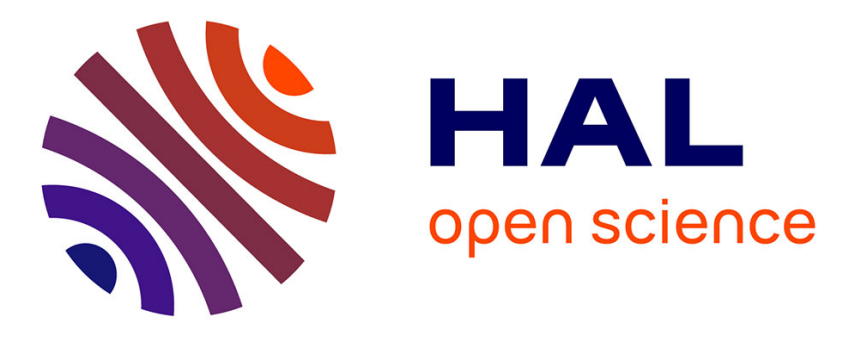

\title{
Simulating the Martian dust cycle with a finite surface dust reservoir
}

\author{
M.A. Kahre, J.R. Murphy, R.M. Haberle, Franck Montmessin, J. Schaeffer
}

\section{To cite this version:}

M.A. Kahre, J.R. Murphy, R.M. Haberle, Franck Montmessin, J. Schaeffer. Simulating the Martian dust cycle with a finite surface dust reservoir. Geophysical Research Letters, 2005, 32, pp.L20204. 10.1029/2005GL023495 . hal-00077313

\section{HAL Id: hal-00077313 https://hal.science/hal-00077313}

Submitted on 31 Jan 2016

HAL is a multi-disciplinary open access archive for the deposit and dissemination of scientific research documents, whether they are published or not. The documents may come from teaching and research institutions in France or abroad, or from public or private research centers.
L'archive ouverte pluridisciplinaire HAL, est destinée au dépôt et à la diffusion de documents scientifiques de niveau recherche, publiés ou non, émanant des établissements d'enseignement et de recherche français ou étrangers, des laboratoires publics ou privés. 


\title{
Simulating the Martian dust cycle with a finite surface dust reservoir
}

\author{
M. A. Kahre and J. R. Murphy \\ Department of Astronomy, New Mexico State University, Las Cruces, New Mexico, USA \\ R. M. Haberle, F. Montmessin, ${ }^{1}$ and J. Schaeffer \\ NASA Ames Research Center, Moffett Field, California, USA
}

Received 17 May 2005; revised 19 August 2005; accepted 13 September 2005; published 26 October 2005.

[1] Multiple year General Circulation Model (GCM) simulations that include a finite surface dust reservoir and an infinite surface dust reservoir are compared. While the infinite dust reservoir simulations produce a highly repeatable annual dust cycle, the finite surface dust reservoir simulations evolve quickly towards a low- dust condition. Once a region is swept clean of available surface dust, it reacquires only small amounts of dust during northern summer but it is repeatedly swept clean during each subsequent dust storm season (southern spring and summer). This argues against a finite dust reservoir as a mechanism for the interannual variability of global dust storms. Additionally, these results suggest that the regions of preferred wind stress lifting are deep dust reservoirs that are not depleted and resupplied on annual or decadal timescales. Therefore, the dust cycle must be closed on much longer timescales, possibly those associated with orbital variations. Citation: Kahre, M. A., J. R. Murphy, R. M. Haberle, F. Montmessin, and J. Schaeffer (2005), Simulating the Martian dust cycle with a finite surface dust reservoir, Geophys. Res. Lett., 32, L20204, doi:10.1029/2005GL023495.

\section{Introduction}

[2] Martian global dust storms are not an annual occurrence [Martin and Zurek, 1993]. This interannual variability is not fully understood. Mars' low-mass atmosphere has a short radiative response time [Goody and Belton, 1967], which makes it difficult for the atmosphere to 'remember' whether or not there was a global dust storm during the previous year. Global scale atmospheric numerical modeling efforts have been successful at reproducing the average annual dust cycle [Newman et al., 2002; Basu et al., 2004]. The interannual variability of global dust storms has been simulated with high friction velocity threshold values [Basu et al., 2004].

[3] While the atmosphere does not retain information from the previous year, hysteresis can remain in the system through the modification of surface properties such as dust availability, albedo, and surface roughness [Pankine and Ingersoll, 2002, 2004].

[4] Published General Circulation Model (GCM) dust cycle simulation efforts have assumed that there was an infinite supply of surface dust planet-wide [Newman et

\footnotetext{
${ }^{1}$ Now at Service d'Aronomie du CNRS, IPSL, Paris, France.
}

al., 2002; Basu et al., 2004]. Interpretation of measured albedo and thermal inertia patterns indicate that this is not likely to be a good assumption for all locations on the surface.

[5] In this work, we study the effects of imposing an initially uniformly distributed finite surface dust reservoir into a version of the NASA Ames Mars General Circulation Model. This model includes a fully interactive dust cycle incorporating lifting, transport, and sedimentation of radiatively active dust. The model is integrated for multiple years to investigate the potential for regions to be swept clean of dust and to determine if such redistribution results in greater interannual variability than occurs under conditions of an inexhaustible surface dust reservoir.

\section{The Ames Mars GCM With an Interactive Dust Cycle}

[6] The NASA/Ames Mars GCM is a three-dimensional gridpoint model of the Martian atmosphere. The model version (1.7.3) used for this study employs the Arakawa C-Grid, with a horizontal resolution of $5^{\circ}$ in latitude and $6^{\circ}$ in longitude. Near the surface, the normalized pressure vertical coordinate system resolution is on the order of tens of meters. Above ten kilometers the vertical resolution is approximately one half of one scale height. The model's lower boundary incorporates MOLA topography and the thermal inertia and albedo fields that are currently used in the LMD model (F. Forget, personal communication). The latter consists of TES data for tropical and middle latitudes [Jakosky et al., 2000] and Viking data for the polar regions [Paige et al., 1994; Paige and Keegan, 1994]. Both the TES and Viking data sets have been corrected for the effects of atmospheric dust [Haberle and Jakosky, 1991]. A manuscript is in preparation describing GCM 1.7.3.

[7] The model includes physical parameterizations for surface dust lifting based upon wind stress [Murphy, 1999; Haberle et al., 2003] and dust devils/convective vortices [Newman et al., 2002].

[8] The dust flux due to winds occurs through the momentum exchange between the atmosphere and the surface. The parameter used to describe this process is the surface stress $(\tau)$, which depends upon the near surface air density, the near surface vertical temperature gradient, and the near surface wind speed and its vertical gradient. The wind lifting prescription is based upon the formulation employed by Westphal et al. [1987] in terrestrial Saharan dust storm simulations with appropriate modifications for Martian conditions [Murphy, 1999; Haberle et al., 2003]. 


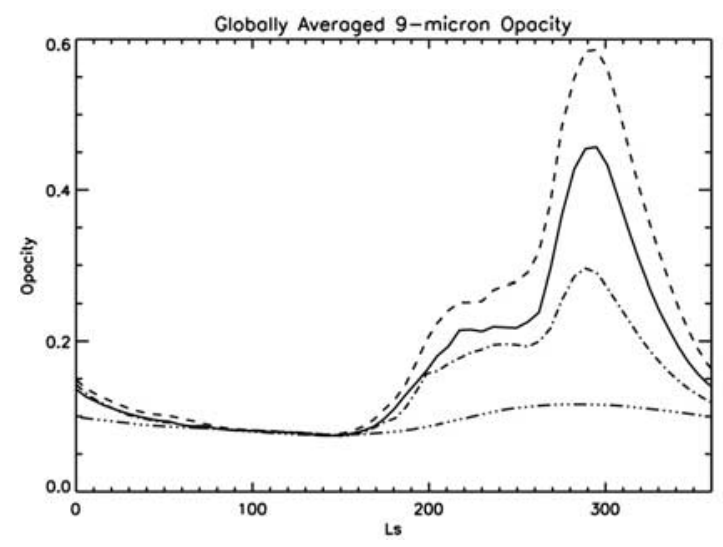

Figure 1. Globally averaged $9 \mu \mathrm{m}$ opacity for $\tau^{*}$ of $0.02 \mathrm{~N} / \mathrm{m}^{2}$ (dashed line), $0.0225 \mathrm{~N} / \mathrm{m}^{2}$ (solid line) and $0.025 \mathrm{~N} / \mathrm{m}^{2}$ (dot-dashed line), and for a dust devil lifting only simulation (dot-dot-dot-dashed line).

The magnitude of the vertical surface dust flux $\left(F_{W}\right)$ is prescribed as:

$$
F_{W}\left(\mathrm{~kg} / \mathrm{m}^{2} / \mathrm{s}\right)=\left(\alpha_{W}\right)\left(2.3 \times 10^{-3}\right)\left(\tau^{2}\right)\left(\frac{\left(\tau-\tau^{*}\right)}{\tau^{*}}\right),
$$

where $\tau^{*}$ is the specified threshold stress required for lifting and $\alpha_{W}$ is a tunable efficiency parameter. Haberle et al. [2003] used this lifting scheme (assuming $\alpha_{W}=1$.) to calculate the surface dust deflation potential with a version of the model that was driven by a spatially and temporally fixed atmospheric dust load. As discussed below, application of this dust lifting prescription results in an annual dust cycle in reasonably good agreement to that simulated by Newman et al. [2002] using a different dust lifting prescription.

[9] The convective vortex (dust devil) surface dust lifting prescription is the threshold independent scheme developed by Newman et al. [2002], and is based upon the magnitude of the vertical heat flux and the depth of the planetary boundary layer.

[10] The dust mass lifted by the wind stress and dust devil parameterizations is partitioned in a log-normal particle size distribution that produces a mean particle radius of approximately $1.5 \mu \mathrm{m}$. This value is consistent with recently derived atmospheric dust particle sizes [Wolff and Clancy, 2003]. The distribution is represented by three particle sizes of radii $0.1 \mu \mathrm{m}, 1.5 \mu \mathrm{m}$ and $10 \mu \mathrm{m}$. These particle sizes account for $3.0 \times 10^{-5} \%, 19.8 \%$, and $80.2 \%$ of the mass and $1 \%, 77 \%$, and $22 \%$ of the optical depth, respectively. These percentages change as the suspended particle size distribution evolves. The opacity of the suspended dust is used in radiative transfer calculations, with a visible-toinfrared opacity ratio equal to 2 .

[11] A set of lifting efficiency values was determined that produced a simulated dust cycle that agrees reasonably well with the observed cycle [Smith, 2004]. For the model resolution and threshold stress specified $\left(0.0225 \mathrm{~N} / \mathrm{m}^{2}\right)$ the optimal wind efficiency factor $\left(\alpha_{W}\right)$ was 0.10 . Plotted in Figure 1 is the seasonal cycle of the globally averaged $9 \mu \mathrm{m}$ opacity for three $\tau^{*}$ cases $(0.020,0.0225$ and
$0.0250 \mathrm{~N} / \mathrm{m}^{2}$ ) and the globally averaged $9 \mu \mathrm{m}$ opacity for a simulation in which only dust devil lifting was employed. The dust devil parameterization provides a background opacity throughout the entire Martian year.

[12] Simulated wind stress dust lifting is primarily confined to middle latitudes (Figure 2) which compares favorably with but is less longitudinally extensive than observed lifting events [Cantor et al., 2001].

[13] Simulated temperatures, zonal winds, mass transport, and vertical atmospheric dust loading (not shown) agree well with observations [Conrath et al., 2000] and previous GCM simulations using fixed atmospheric dust loading with comparable global opacities [Haberle et al., 1993].

[14] Finally, the model was run with the most recent wind stress dust lifting prescription developed by Newman et al. [2005]. Using the a stress threshold of $0.0225 \mathrm{~N} / \mathrm{m}^{2}$, the Newman scheme was tuned to produce a simulated dust cycle that mimics the annual dust cycle simulated with our lifting scheme. The resulting efficiency parameter value of $9 \times 10^{-6}$ is a factor of almost four greater than the value used in radiatively active dust simulations (with a stress threshold of $0.01 \mathrm{~N} / \mathrm{m}^{2}$ ) by Newman et al. [2005]. The dust lifting rate produced with the tuned Newman scheme and the scheme described above are very similar within the wind stress range of 0 . to $0.05 \mathrm{~N} / \mathrm{m}^{2}$. We chose to use the prescription described above because we understand the sensitivities associated with this scheme more completely.

\section{Simulation Results}

[15] Several multi-year simulations of the Ames GCM were run with the dust lifting parameterizations described above. The first 10-year simulation included an infinite planet-wide supply of surface dust and a stress threshold of $0.0225 \mathrm{~N} / \mathrm{m}^{2}$. The maximum net deflation $(280 \mu \mathrm{m})$ during one year of this simulation was located in the Hellas region. Multi-year simulations with initial finite surface dust depths of 25,100 , and $200 \mu \mathrm{m}$ with a wind stress threshold

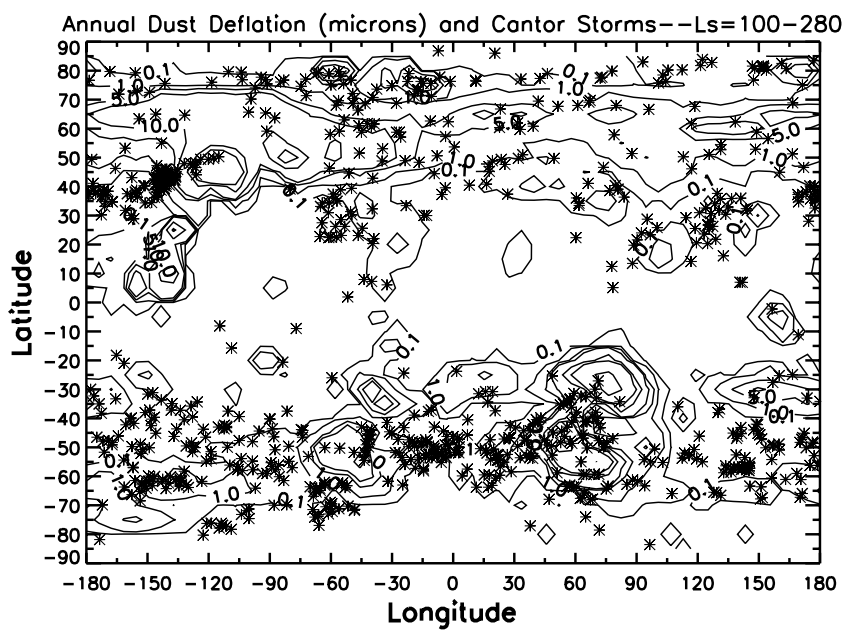

Figure 2. Contours: deflation due to winds $\left(\mathrm{L}_{s}=100-\right.$ 280; contour levels are $0.1,1.0,10,100$, and $100 \mu \mathrm{m}$. Asterisks: MOC observations of dust activity during this season. 


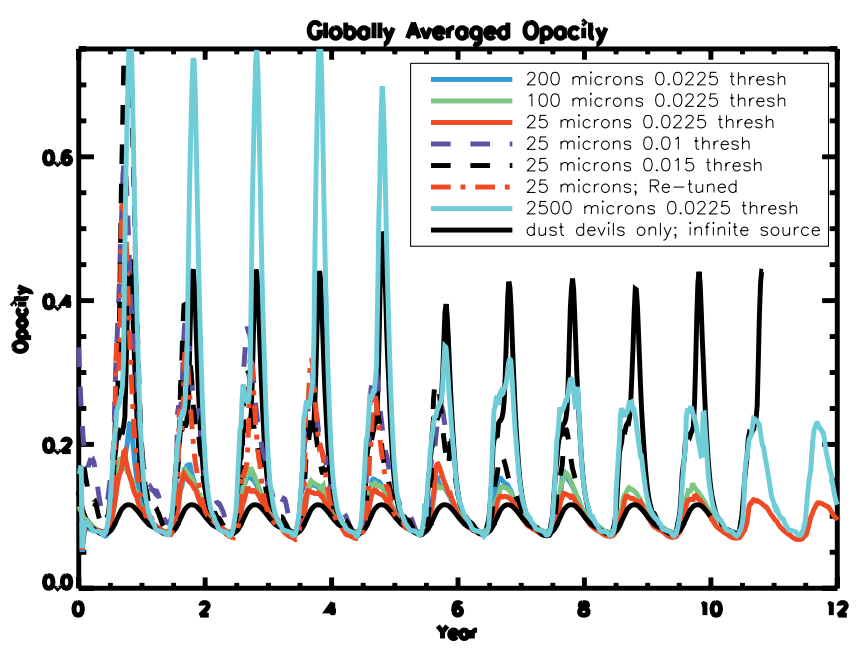

Figure 3. Multi-year globally averaged $9 \mu \mathrm{m}$ opacity for the infinite surface dust simulation (black solid) the finite surface dust simulations (colored solid and dashed lines).

of $0.0225 \mathrm{~N} / \mathrm{m}^{2}$ were designed to ensure that a varying number of regions would be depleted during the first year of simulation. Additionally, two simulations were initiated with lower thresholds (i.e., 0.01 and $0.015 \mathrm{~N} / \mathrm{m}^{2}$ ) and an initial finite surface dust depth of $25 \mu \mathrm{m}$; these simulations were tuned to produce a global dust event during the first year of simulation (i.e., globally averaged $9 \mu \mathrm{m}$ opacities exceeding 1.5). One simulation was initiated with the surface dust spatial distribution that existed at the end of the tenth simulated year of the $25 \mu \mathrm{m} 0.0225 \mathrm{~N} / \mathrm{m}^{2}$ simulation described above. This simulation included an increased efficiency factor and was designed to force secondary source regions to become primary source regions to explore the possibility that these regions could be resupplied cyclically. Finally, one simulation was initiated with $2500 \mu \mathrm{m}$ of surface dust globally, a threshold of $0.0225 \mathrm{~N} / \mathrm{m}^{2}$, and an efficiency factor that produces peak opacities consistent with a global dust storm year. This simulation was designed to not allow any region to be depleted of surface dust until the 6 th simulated year.

\subsection{Infinite Source}

[16] In the multi-year infinite source simulation, the globally averaged $9 \mu \mathrm{m}$ opacity is highly repeatable from one year to the next (Figure 3). The simulated annual peak of the globally averaged $9 \mu \mathrm{m}$ opacity occurs between $\mathrm{L}_{s}=$ 289 and $\mathrm{L}_{s}=303$; the magnitude of the peak varies from 0.4 to 0.5 . The global quantity and latitudinal pattern of suspended dust are consistent with $9 \mu \mathrm{m}$ opacities derived from TES spectra during mapping year 1. However, the simulated peak globally averaged $9 \mu \mathrm{m}$ opacity occurs several tens of degrees of $\mathrm{L}_{s}$ later than the observed peak [Smith, 2004].

[17] In this simulation, the total dust lifted annually by dust devils is comparable in magnitude to the total dust lifted annually by wind stress lifting. Dust devils provide a spatially and temporally extensive source of dust lifting that does not result in the removal of more than $20 \mu \mathrm{m}$ of surface dust from any one location during one simulated year. Wind stress lifting results in the removal of more substantial quantities of dust from localized regions.

[18] Three distinct regions of preferred wind stress lifting appear in the infinite source simulation: the northeast interior slope of Hellas, the southwest interior slope of Hellas, and a region northwest of Tharsis. In these locations, the annual net deflation exceeds $50 \mu \mathrm{m}$. Regions of maximum deposition include the center of Hellas and locations adjacent to the maximum deflation region northwest of Tharsis. The net deposition in these regions exceeds $25 \mu \mathrm{m}$. The center of Syris Major experiences annual net deflation, but only poor correlations exist elsewhere between annual net deflation/deposition and albedo or thermal inertia.

\subsection{Finite Source}

[19] In all of the multi-year finite source simulations, once at least one region is depleted of surface dust, the annual peak opacity is less in subsequent simulated years (Figure 3 ). The peaks of the globally averaged opacity in the finite surface dust simulation occur earlier in the year (between $\mathrm{L}_{s}=227$ and $\mathrm{L}_{s}=265$ ) than in the infinite case, which is in better agreement with observations [Smith, 2004].

[20] The annual globally averaged peak $9 \mu \mathrm{m}$ opacity declines from one simulated year to the next until a low-dust steady state condition is reached in which the peak annual $9 \mu \mathrm{m}$ opacity does not exceed 0.2 . Simulations initiated with a global dust event via low stress thresholds (i.e., 0.01 and $0.015 \mathrm{~N} / \mathrm{m}^{2}$ ) and large initial surface dust reservoirs (i.e., $2500 \mu \mathrm{m})$ take longer to evolve to the low global dust loading condition and do not produce subsequent dust storm years once regions become swept clean.

[21] As the finite dust simulations evolve beyond the point at which regions become depleted, the relative contribution of dust devils to the total amount of dust lifted increases. By the second year in the $0.0225 \mathrm{~N} / \mathrm{m}^{2}$ threshold simulations, the amount of dust lifted annually by dust devils is a factor of 2 greater than the amount of dust lifted annually by wind stress lifting. The lower stress threshold simulations are dominated by wind stress lifting throughout the duration of the simulations, but the relative contribution of dust devils increases as the simulations evolve.

\section{Discussion}

[22] Interannual variability of the global dust cycle does not occur in any of the finite surface dust simulations. The surface dust reservoir continues to evolve throughout the entire duration of the finite surface dust simulations. With few exceptions, regions that experience a net deposition of dust during the first year of simulation continue to accumulate dust throughout the simulation. Similarly, regions that experience net deflation of dust during the first simulated year continue to lose dust until they are swept clean. A few regions undergo a slight net annual deflation early in the simulation and then become net deflation regions as the simulation continues. However, no region experiences cyclic net deflation and net deposition on multi-year timescales. The enhanced efficiency factor finite source simulation initiated with the surface dust field 
from the end of the tenth year of the $25 \mu \mathrm{m} 0.0225 \mathrm{~N} / \mathrm{m}^{2}$ simulation also evolves quickly towards a low dust state, suggesting that source regions in this simulation are not resupplied. The finite reservoir simulation that included a large initial surface dust reservoir (i.e., $2500 \mu \mathrm{m}$ ) also evolves to a low-dust state once regions are finally swept clean. This suggests that the results presented here are independent of the initial surface dust inventory.

[23] As the finite dust simulations evolve towards a lowatmospheric dust situation, dust devils become a more important dust lifting mechanism than wind stress lifting. As preferred wind stress lifting regions become depleted of available surface dust, the only resupplying mechanism for these regions becomes dust devils. Swept clean regions accumulate small amounts (i.e., a couple of microns) of surface dust during northern summer due to the globalscale deposition of dust injected into the atmosphere by dust devil lifting. However, these small dust accumulations are quickly swept away annually by wind stress lifting during late southern spring.

[24] In order for the finite surface dust source to cause interannual variability, there must be more dust available during some years than others in swept clean regions. Specifically, these fully depleted regions must gain dust and keep it throughout the dust storm season during some martian years. This is not the case in these simulations.

[25] If these simulated swept clean regions were fully depleted of dust on Mars, one would expect to observe little if any dust storm activity in these regions. However, dust events have been observed in abundance in these regions [Cantor et al., 2001]. This would not be a problem if there is a supply of dust in these regions that has not yet been exhausted. Based on the maximum depth of dust deflated in one simulated year with an infinite surface dust reservoir $(280 \mu \mathrm{m})$, a dust reservoir in this location of 280 meters could be removed in one million years.

[26] Another potential solution to the inconsistency discussed above could be that there is a problem with the manner in which the dust cycle is simulated in the GCM. It is possible that physical processes (e.g., wind stress dust lifting) are implemented incorrectly or incompletely. It is also possible that there are vital physical processes that are currently not included. Such a process would need to encourage dust to accumulate in preferred lifting regions. This accumulation would need to be large enough so that these regions could be substantial dust sources in subsequent years. Both the wind stress lifting threshold and the surface roughness are temporally and spatially fixed in the simulations discussed in this paper. Pankine and Ingersoll [2002, 2004] discuss the negative feedback between increasing the surface roughness and decreasing the stress threshold stress. For their theory to be correct, however, surface roughness must be altered significantly on annual timescales. The maximum deposition during one year in the infinite surface dust simulation was $90 \mu \mathrm{m}$. It is unclear if this amount of dust deposition would have a significant impact upon the lifting threshold on an annual basis.

[27] Feedbacks between dust deposition/removal and albedo and thermal inertia are also not included. Changing the albedo alters the thermal state of the atmosphere and the depth of the planetary boundary layer [Kahre et al., 2005].
Thus, including these feedbacks could potentially alter the results presented here. These are interesting questions that deserve further investigation.

\section{Conclusions}

[28] The three main conclusion of this work are the following:

[29] 1. Imposing a finite dust reservoir into GCM simulations of the Martian dust cycle does not provide a mechanism for simulating the interannual variability of global dust storms. Regions that become swept clean do not subsequently accumulate dust; therefore, they never again become significant source regions. In the context of this model, it is clear that additional physical mechanisms are required for the interannual variability of the annual dust cycle to be realized.

[30] 2. Since the implementation of a finite surface dust reservoir does not result in a realistic simulated dust cycle, the dust reservoirs in preferred wind stress lifting regions are likely to be deep and have not yet been depleted. Based on simulated net deflation rates, these dust reservoirs must be hundreds of meters deep to survive for one million years.

[31] 3. Based on these model results, the dust cycle cannot be closed on annual or decadal timescales. Therefore, the dust cycle must be closed on much longer timescales, possibly those associated with orbital variations.

[32] Acknowledgments. We would like to thank Phil Christensen and Ron Greeley for helpful discussions regarding this work. Funding was received from the NASA Graduate Student Researchers Program and NASA's Planetary Atmospheres Program (NASA/NAG5-12123).

\section{References}

Basu, S., M. I. Richardson, and R. J. Wilson (2004), Simulation of the Martian dust cycle with the GFDL Mars GCM, J. Geophys. Res., 109, E11006, doi:10.1029/2004JE002243.

Cantor, B. A., P. B. James, M. Caplinger, and M. J. Wolff (2001), Martian dust storms: 1999 Mars Orbiter camera observations, J. Geophys. Res., 106, 23,653-23,687.

Conrath, B. J., J. C. Pearl, M. D. Smith, W. C. Maguire, P. R. Christensen, S. Dason, and M. S. Kaelberer (2000), Mars Global Surveyor Thermal Emission Spectrometer (TES) observations: Atmospheric temperatures during aerobraking and science phasing, J. Geophys. Res., 105, 95099519 .

Goody, R., and M. J. S. Belton (1967), Radiative relaxation times for Mars: A discussion of Martian atmospheric dynamics, Planet. Space Sci., 15, 247-256.

Haberle, R. M., and B. M. Jakosky (1991), Atmospheric effects on the remote determination of thermal inertia of Mars, Icarus, 90, 187-204.

Haberle, R. M., J. B. Pollack, J. R. Barnes, R. W. Zurek, C. B. Leovy, J. R. Murphy, H. Lee, and J. Schaeffer (1993), Mars atmospheric dynamics as simulated by the NASA AMES General Circulation Model: 1. The zonal-mean circulation, J. Geophys. Res., 98, 3093-3123.

Haberle, R. M., J. R. Murphy, and J. Schaeffer (2003), Orbital change experiments with a Mars general circulation mode, Icarus, 161, 66-89.

Jakosky, B. M., M. T. Mellon, H. H. Kieffer, P. R. Christensen, V. E. Stacy, and S. W. Lee (2000), The thermal inertia of Mars from the Mars Global Surveyor Thermal Emission Spectrometer, J. Geophys. Res., $105,9643-9652$.

Kahre, M. A., J. R. Murphy, N. J. Chanover, J. L. Africano, L. C. Roberts, and P. W. Kervin (2005), Observing the Martian Surface Albedo Pattern: Comparing the AEOS and TES Data Sets, Icarus, in press.

Martin, L. J., and R. W. Zurek (1993), Interannual variability of planetencircling dust storms on Mars, J. Geophys. Res., 98, 3221-3246.

Murphy, J. R. (1999), The Martian atmospheric dust cycle: Insights from numerical model simulations, paper presented at Fifth International Conference on Mars, Lunar and Planet. Inst., Pasadena, Calif.

Newman, C. E., S. R. Lewis, P. L. Read, and F. Forget (2002), Modeling the Martian dust cycle 2. Multiannual radiatively active dust 
transport simulations, J. Geophys. Res., 107(E12), 5124, doi:10.1029/ 2002JE001920.

Newman, C. E., S. R. Lewis, and P. L. Read (2005), The atmospheric circulation and dust activity in different orbital epochs on Mars, Icarus, $174,135-160$.

Paige, D. A., and K. D. Keegan (1994), Thermal and albedo mapping of the polar regios of Mars using Viking thermal mapper observations: 2 . South polar region, J. Geophys. Res., 99, 25,993-26,013.

Paige, D. A., J. E. Bachman, and K. D. Keegan (1994), Thermal and albedo mapping of the polar regions of Mars using Viking thermal mapper observations: 1. North polar region, J. Geophys. Res., 99, 25,959$25,992$.

Pankine, A. A., and A. P. Ingersoll (2002), Interannual variability of Martian global dust storms - Simulations with a low-order model of the general circulation, Icarus, 155, 299-323.

Pankine, A. A., and A. P. Ingersoll (2004), Interannual variability of Mars global dust storms: An example of self-organized criticality?, Icarus, $170,514-518$
Smith, M. D. (2004), Interannual variability in TES atmospheric observations of Mars during 1999-2003, Icarus, 167, 148-165.

Westphal, D. L., O. B. Toon, and T. N. Carlson (1987), A two-dimensional numerical investigation of the dynamics and microphysics of Saharan dust storms, J. Geophys. Res., 92, 2039-3027.

Wolff, M. J., and R. T. Clancy (2003), Constraints on the size of Martian aerosols from Thermal Emission Spectrometer observations, J. Geophys. Res., 108(E9), 5097, doi:10.1029/2003JE002057.

R. M. Haberle and J. Schaeffer, NASA Ames Research Center, Moffett Field, CA 94035-1000, USA.

M. A. Kahre and J. R. Murphy, Department of Astronomy, New Mexico State University, MSC 4500/Box 30001, Las Cruces, NM 88003, USA. (kahrema@nmsu.edu)

F. Montmessin, Service d'Aronomie du CNRS/IPSL, Tour 45-46 (bo te 102), Univ. Pierre et Marie Curie, 4, place Jussieu, F-75252 Paris Cedex 05, France. 